\title{
ON THE VOLUME OF NODAL SETS FOR EIGENFUNCTIONS OF THE LAPLACIAN ON THE TORUS
}

\author{
ZEÉV RUDNICK AND IGOR WIGMAN
}

\begin{abstract}
We study the volume of nodal sets for eigenfunctions of the Laplacian on the standard torus in two or more dimensions. We consider a sequence of eigenvalues $4 \pi^{2} E$ with growing multiplicity $\mathcal{N} \rightarrow \infty$, and compute the expectation and variance of the volume of the nodal set with respect to a Gaussian probability measure on the eigenspaces. We show that the expected volume of the nodal set is const $\sqrt{E}$. Our main result is that the variance of the volume normalized by $\sqrt{E}$ is bounded by $O(1 / \sqrt{\mathcal{N}})$, so that the normalized volume has vanishing fluctuations as we increase the dimension of the eigenspace.
\end{abstract}

\section{INTRODUCTION}

The nodal set of a function on a manifold is the set of points where it vanishes. Nodal sets for eigenfunctions of the Laplacian on a smooth, compact Riemannian manifold have been studied intensively for some time now. For instance, it is known [6] that except for a subset of lower dimension, the nodal sets of eigenfunctions are smooth manifolds of codimension one in the ambient manifold. In particular one can define their hypersurface volume (in two dimensions this is the length). A conjecture of Yau is that the volume of the nodal set is bounded above and below by constant multiples of square root of the Laplace eigenvalue. Yau's conjecture was proven for real-analytic metrics by Donnelly and Fefferman [7]. The lower bound in the case of smooth surfaces is due to Brüning [4], see also [5] for planar domains.

In this paper we study the volume of nodal sets for eigenfunctions of the Laplacian on the standard flat torus $\mathbb{T}^{d}=\mathbb{R}^{d} / \mathbb{Z}^{d}, d \geq 2$. We write the eigenvalue equation as $\Delta f=-4 \pi^{2} E f$, where $E \geq 0$ is an integer. The eigenvalues on the torus always have multiplicities, with the dimension $\mathcal{N}=\mathcal{N}(E)$ of an eigenspace corresponding to eigenvalue $4 \pi^{2} E$ being the number of integer vectors $\lambda \in \mathbb{Z}^{d}$ so that $|\lambda|^{2}=E$. In dimension $d \geq 5$ this number grows as $E \rightarrow \infty$ roughly as $E^{\frac{d}{2}-1}$, but for small values of $d$, particularly for $d=2$, the behaviour is more erratic, and depends on the prime decomposition of $E$.

We will consider random eigenfunctions on the torus, that is random linear combinations

$$
f(x)=\frac{1}{\sqrt{2 \mathcal{N}}} \sum_{\lambda \in \mathbb{Z}^{d}:|\lambda|^{2}=E} b_{\lambda} \cos 2 \pi\langle\lambda, x\rangle-c_{\lambda} \sin 2 \pi\langle\lambda, x\rangle
$$

Date: September 23, 2007.

Z.R. was supported by the Israel Science Foundation (grant No. 925/06). I.W was supported by CRM analysis laboratory fellowship. 
with $b_{\lambda}, c_{\lambda} \sim N(0,1)$ real Gaussians of zero mean and variance 1 which are independent save for the relations $b_{-\lambda}=b_{\lambda}, c_{-\lambda}=-c_{\lambda}$. Let $\mathcal{E}=\mathcal{E}_{E}$ be the eigenspace associated to the eigenvalue $4 \pi^{2} E$ (i.e. the space of functions of form (1.1) ). We denote by $\mathbb{E}(\bullet)$ the expected value of the quantity $\bullet$ in this ensemble. For instance, the expected amplitude of $f$ is $\mathbb{E}\left(|f(x)|^{2}\right)=1$.

Denote by $Z(f)$ the volume of the nodal set of an eigenfunction (1.1). Our first result, Proposition 4.1, is that the expected value of $Z$ is

$$
\mathbb{E}(Z)=\text { const } \cdot \sqrt{E}
$$

for a certain constant depending only on the dimension $d$. This is of course consistent with the bounds of Donnelly and Fefferman [7].

Our main result, Theorem [6.1, is that the variance of the normalized volume $Z / \sqrt{E}$ is bounded by

$$
\operatorname{Var}\left(\frac{Z}{\sqrt{E}}\right) \ll \frac{1}{\sqrt{\mathcal{N}}}, \quad \text { as } \mathcal{N} \rightarrow \infty .
$$

(We believe that the correct upper bound for the variance is $O(1 / \mathcal{N})$ ). Thus the fluctuations of $Z(f) / \sqrt{E}$ around its mean value die out as the multiplicity $\mathcal{N}$ tends to $\infty$. Note however that $Z(f) / \sqrt{E}$ is not asymptotically constant; for instance, if $E=d m^{2}$ then for the eigenfunction $f(x)=\prod_{j=1}^{d} \sin 2 \pi m x_{j}$ we have $Z(f) / \sqrt{E}=2 \sqrt{d}$ while if $E=m^{2}$ then for the eigenfunction $f(x)=\sin 2 \pi m x_{1}$ we have $Z(f) / \sqrt{E}=2$.

Theorem 6.1 can be viewed as lending support to the expectation 1 that for eigenfunctions on negatively curved manifolds, which are believed to behave similarly to random waves [2], the volumes of nodal sets, normalized by the square-root of the eigenvalue, do tend to a limiting value. See [12] for some work on the complexified nodal set of eigenfunctions in this context.

Previous work in this vein is due to Bérard [1, who computed the expected surface measure of the nodal set for eigenfunctions of the Laplacian on spheres. Neuheisel [10] also worked on the sphere and gave an upper bound for the variance. Berry [3] computed the expected length of nodal lines for isotropic, monochromatic random waves in the plane, which are eigenfunctions of the Laplacian with eigenvalue $E$. He found that the expected length (per unit area) is again of size about $\sqrt{E}$ and argued that the variance should be of order $\log E$.

More recently, F. Oravecz and ourselves have investigated a different characteristic of the nodal set of eigenfunctions on the torus, namely the Leray nodal measure [11, and have succeeded in obtaining the precise asymptotics of the variance of the Leray measure as $\mathcal{N} \rightarrow \infty$.

1.1. Plan of the paper. We employ a version of the Kac-Rice formula for the volume of the nodal set, which using the Dirac delta function can be written as

$$
Z(f)=\int_{\mathbb{T}^{d}} \delta(f(x))|\nabla f(x)| d x,
$$

see $\oint 3$ for the rigorous version. To compute the expected value of $Z$ is then a simple matter once we find that $f(x)$ and $\partial f / \partial x_{j}$ are independent Gaussians. This is done in $\S$, In $\S$ 5 we derive a formula for the second moment of $Z$,

\footnotetext{
${ }^{1}$ We thank Steve Zelditch for a discussion of this.
} 
which requires knowing the covariance structure of the $2 d+2$-dimensional Gaussian vector $v(x, y)=(f(x), f(y), \nabla f(x), \nabla f(y))$. That $v(x, y)$ is indeed a non-degenerate $2 d+2$ dimensional Gaussian is verified in the Appendix. As a result, we find that $\mathbb{E}\left(Z^{2}\right)=\int_{\mathbb{T}^{d}} K(z) d z$, with

$$
K(z)=\frac{1}{\sqrt{1-u(z)^{2}}} \int_{\mathbb{R}^{2 d}}\left\|v_{1}\right\|\left\|v_{2}\right\| \frac{\exp \left(-\frac{1}{2} v \Omega(z)^{-1} v^{T}\right)}{\sqrt{\operatorname{det} \Omega(z)}} \frac{d v}{(2 \pi)^{d+1}},
$$

where $u(z)=\mathbb{E}(f(x) f(x+z))$ is the two-point function of the ensemble, and where $\Omega(z)$ is a certain positive definite $2 d \times 2 d$ matrix which enters into the covariance structure of the Gaussian vector $v(x, y)$. In section $\sqrt[6]{6}$, which is the heart of the paper, we bound the variance of $Z$.

\section{THE MODEL: RANDOM EIGENFUNCTIONS ON THE TORUS}

2.1. Random eigenfunctions. We consider non-constant eigenfunctions of the Laplacian on the standard flat torus $\mathbb{T}^{d}=\mathbb{R}^{d} / \mathbb{Z}^{d}$. The solutions of the eigenvalue equation

$$
\Delta \psi+4 \pi^{2} E \psi=0, \quad E \neq 0,
$$

form a finite dimensional vector space $\mathcal{E}=\mathcal{E}_{E}$, having as a basis the exponentials $e^{2 \pi i\langle\lambda, x\rangle}$, for $\lambda$ in the frequency set

$$
\Lambda=\Lambda_{E}=\left\{\lambda \in \mathbb{Z}^{d},|\lambda|^{2}=E\right\} .
$$

We define an ensemble of Gaussian random functions $f \in \mathcal{E}$ by

$$
f(x)=\frac{1}{\sqrt{2 \mathcal{N}}} \sum_{\lambda \in \Lambda} b_{\lambda} \cos 2 \pi\langle\lambda, x\rangle-c_{\lambda} \sin 2 \pi\langle\lambda, x\rangle
$$

with $b_{\lambda}, c_{\lambda} \sim N(0,1)$ real Gaussians of zero mean and variance 1 which are independent save for the relations $b_{-\lambda}=b_{\lambda}, c_{-\lambda}=-c_{\lambda}$. Thus we can rewrite

$$
f(x)=\sqrt{\frac{2}{\mathcal{N}}} \sum_{\lambda \in \Lambda / \pm} b_{\lambda} \cos 2 \pi\langle\lambda, x\rangle-c_{\lambda} \sin 2 \pi\langle\lambda, x\rangle
$$

where now only independent random variables appear. With our normalization, we have $\mathbb{E}\left(|f(x)|^{2}\right)=1$ for all $x \in \mathbb{T}^{d}$.

Definition 2.1. An eigenfunction $f \in \mathcal{E}$ is singular if $\exists x \in \mathbb{T}^{d}$ with $f(x)=0$ and $\nabla f(x)=\overrightarrow{0}$. An eigenfunction $f \in \mathcal{E}$ is nonsingular if $\nabla f \neq \overrightarrow{0}$ on the nodal set.

Lemma 2.2 ([1], Lemma 2.3). The set of singular eigenfunctions has codimension at least 1 in $\mathcal{E}$, and so has measure zero in $\mathcal{E}$.

2.2. Properties of the frequency set. The dimension $\mathcal{N}=\operatorname{dim} \mathcal{E}$ is the number of the frequencies in $\Lambda$, which is the number of ways of expressing $E$ as a sum of $d$ integer squares. For $d \geq 5$ this grows roughly as $E^{d / 2-1}$ as $E \rightarrow \infty$. For $d \leq 4$ the dimension of the eigenspace need not grow with $E$. For instance, for $d=2, \mathcal{N}$ is given in terms of the prime decomposition of $E$ as follows: If $E=2^{\alpha} \prod_{j} p_{j}^{\beta_{j}} \prod_{k} q_{k}^{2 \gamma_{k}}$ where $p_{j} \equiv 1 \bmod 4$ and $q_{k} \equiv 3$ mod 4 are odd primes, $\alpha, \beta_{j}, \gamma_{k} \geq$ are integers, then $\mathcal{N}=4 \prod_{j}\left(\beta_{j}+1\right)$, and 
otherwise $E$ is not a sum of two squares and $\mathcal{N}=0$. On average (over integers which are sums of two squares) the dimension is const $\cdot \sqrt{\log E}$.

The frequency set $\Lambda$ is invariant under the group $W_{d}$ of signed permutations, consisting of coordinate permutations and sign-change of any coordinate, e.g. $\left(\lambda_{1}, \lambda_{2}\right) \mapsto\left(-\lambda_{1}, \lambda_{2}\right)$ (for $d=2$ ). In particular $\Lambda$ is symmetric under $\lambda \mapsto-\lambda$ and since $0 \notin \Lambda$, we find $\mathcal{N}$ is even. We write $\Lambda / \pm$ to denote representatives of the equivalence class of $\Lambda$ under $\lambda \mapsto-\lambda$.

We will need some simple properties of $\Lambda$ :

Lemma 2.3. For any subset $\mathcal{O} \subset \Lambda$ which is invariant under the group $W_{d}$, we have

$$
\frac{1}{|\mathcal{O}|} \sum_{\lambda \in \mathcal{O}} \lambda_{j} \lambda_{k}=\frac{E}{d} \cdot \delta_{j, k}
$$

Moreover for any $C \in \mathbb{R}^{d}$,

$$
\frac{1}{|\mathcal{O}|} \sum_{\lambda \in \mathcal{O}}\langle C, \lambda\rangle^{2}=\frac{E}{d}|C|^{2} .
$$

Proof. For $i \neq j$ use the symmetry of $\mathcal{O}$ under the sign change of the $i$-th coordinate to change variables and deduce that the LHS of (2.2) vanishes. For $i=j$ note that the $\operatorname{sum} \sum_{\lambda \in \mathcal{O}} \lambda_{i}^{2}$ is independent of $i$ since $\mathcal{O}$ is symmetric under permutations; hence we may average the RHS over $i$ to find that

$$
\sum_{\lambda \in \mathcal{O}} \lambda_{i}^{2}=\frac{1}{d} \sum_{i=1}^{d} \sum_{\lambda \in \mathcal{O}} \lambda_{i}^{2}=\frac{1}{d} \sum_{\lambda \in \mathcal{O}}\|\lambda\|^{2}=\frac{|\mathcal{O}| E}{d},
$$

proving (2.2). To prove (2.3) we expand $\langle C, \lambda\rangle^{2}=\sum_{j, k=1}^{d} c_{j} c_{k} \lambda_{j} \lambda_{k}$ and use (2.2).

Note that (2.3) implies that the frequency set $\Lambda$ spans $\mathbb{R}^{d}$.

2.3. The two point function. The two-point function of the ensemble is

$$
u(z):=\mathbb{E}(f(x+z) f(x))=\frac{2}{\mathcal{N}} \sum_{\lambda \in \Lambda / \pm} \cos 2 \pi\langle\lambda, z\rangle .
$$

The two-point function clearly satisfies $|u(z)| \leq 1$. We will need to know some of its basic properties, proved in [11], which we summarize as:

Proposition 2.4. The two point function satisfies

(1) There are only finitely many points $x \in \mathbb{T}^{d}$ where $u(x)= \pm 1$.

(2) The mean square of $u$ is $\int_{\mathbb{T}^{d}} u^{2}=\frac{1}{\mathcal{N}}$.

(3) The mean fourth power of $u$ is bounded by $2 \int_{\mathbb{T}^{d}} u^{4} \ll 1 / \mathcal{N}$.

(4) The kernel $1 / \sqrt{1-u^{2}}$ is integrable on $\mathbb{T}^{d}$.

Part 1 follows from [11, lemma 2.2], part 3 is [11, Proposition 7.1], and part 4 is [11, Lemma 5.3].

\footnotetext{
${ }^{2}$ Except possibly in dimensions $d=3,4$ we have a better bound in [11] of $o(1 / \mathcal{N})$, though we have no use for this finer information in this paper.
} 


\section{A formula for the Volume of the NODAL SET}

Let $\chi$ be the indicator function of the interval $[-1,1]$. We define for $\epsilon>0$

$$
Z_{\epsilon}(f):=\frac{1}{2 \epsilon} \int_{\mathbb{T}^{d}} \chi\left(\frac{f(x)}{\epsilon}\right)|\nabla f(x)| d x .
$$

Lemma 3.1. Suppose that $f \in \mathcal{E}$ is non-singular. Then

$$
\operatorname{vol}\left(f^{-1}(0)\right)=\lim _{\epsilon \rightarrow 0} Z_{\epsilon}(f) .
$$

Proof. By the co-area formula [8], for $f$ smooth and $\phi$ integrable, we have

$$
\int_{\mathbb{T}^{d}} \phi(x)|\nabla f(x)| d x=\int_{-\infty}^{\infty}\left(\int_{f^{-1}(s)} \phi(x) d x\right) d s .
$$

Taking $\phi(x):=\frac{1}{2 \epsilon} \chi\left(\frac{f(x)}{\epsilon}\right)$, which is constant on the level sets $f^{-1}(s)$ gives

$$
Z_{\epsilon}(f)=\frac{1}{2 \epsilon} \int_{-\epsilon}^{\epsilon} \operatorname{vol}\left(f^{-1}(s)\right) d s .
$$

Now if $f$ is non-singular then $s \mapsto \operatorname{vol}\left(f^{-1}(s)\right)$ is continuous at $s=0$ and so by the fundamental theorem of calculus,

$$
\lim _{\epsilon \rightarrow 0} \frac{1}{2 \epsilon} \int_{-\epsilon}^{\epsilon} \operatorname{vol}\left(f^{-1}(s)\right) d s=\operatorname{vol}\left(f^{-1}(0)\right) .
$$

Thus $\lim _{\epsilon \rightarrow 0} Z_{\epsilon}(f)=\operatorname{vol}\left(f^{-1}(0)\right)$ as claimed.

Lemma 3.2. For all $f \in \mathcal{E}$ we have

$$
Z_{\epsilon}(f) \leq 6 d \sqrt{E}
$$

We begin with the one variable case which we state as a separate lemma (cf [9, Lemma 2]):

Lemma 3.3. Let $g(t)$ be a trigonometric polynomial of degree at most $M$. Then for all $\epsilon>0$ we have

$$
\frac{1}{2 \epsilon} \int_{\{t:|g(t)| \leq \epsilon\}}\left|g^{\prime}(t)\right| d t \leq 6 M .
$$

Proof. We partition the set $\{t:|g(t)| \leq \epsilon\} \subseteq[0,1]$ into a union of maximal closed intervals $\left[a_{k}, b_{k}\right]$ (with $a_{k}<b_{k}$ ), disjoint except perhaps for common edges, such that on each such interval $g^{\prime}$ has constant sign, that is either $g^{\prime} \geq 0$ or $g^{\prime} \leq 0$. If $g^{\prime} \geq 0$ on $\left[a_{k}, b_{k}\right]$ then either $g\left(a_{k}\right)=-\epsilon$ or $g^{\prime}\left(a_{k}\right)=0$ and $a_{k}$ is a local minimum for $g$, and $g\left(b_{k}\right) \leq+\epsilon$. If $g^{\prime} \leq 0$ on $\left[a_{k}, b_{k}\right]$ then either $g\left(a_{k}\right)=+\epsilon$ or $g^{\prime}\left(a_{k}\right)=0$ and $a_{k}$ is a local maximum for $g$, and $g\left(b_{k}\right) \geq-\epsilon$.

If $g^{\prime} \geq 0$ on $\left[a_{k}, b_{k}\right]$ then

$$
\int_{a_{k}}^{b_{k}}\left|g^{\prime}(t)\right| d t=\int_{a_{k}}^{b_{k}} g^{\prime}(t) d t=g\left(b_{k}\right)-g\left(a_{k}\right) \leq 2 \epsilon,
$$

while if $g^{\prime} \leq 0$ on $\left[a_{k}, b_{k}\right]$ then

$$
\int_{a_{k}}^{b_{k}}\left|g^{\prime}(t)\right| d t=\int_{a_{k}}^{b_{k}}-g^{\prime}(t) d t=g\left(a_{k}\right)-g\left(b_{k}\right) \leq 2 \epsilon .
$$


Thus the total integral is bounded by the number $\nu$ of intervals $\left[a_{k}, b_{k}\right]$ :

$$
\frac{1}{2 \epsilon} \int_{\{t:|g(t)| \leq \epsilon\}}\left|g^{\prime}(t)\right| d t \leq \nu
$$

Now the number of intervals is bounded by the number of $a$ 's for which $g(a)= \pm \epsilon$ plus the number of $a$ 's for which $g^{\prime}(a)=0$. Since both $g$ and $g^{\prime}$ are trigonometric polynomials of degree $\leq M$, the number of such intervals is therefore $3 \cdot 2 M=6 M$. This gives the required bound.

We now prove Lemma 3.2 by reduction to the one-dimensional case.

Proof. Since $|\nabla f| \leq \sum_{j=1}^{d}\left|\frac{\partial f}{\partial x_{j}}\right|$ we have

$$
Z_{\epsilon}(f) \leq \sum_{j=1}^{d} \frac{1}{2 \epsilon} \int_{\mathbb{T}^{d}} \chi\left(\frac{f(x)}{\epsilon}\right)\left|\frac{\partial f}{\partial x_{j}}\right| d x
$$

and we will bound each term. Taking $j=1$, we have

$$
\frac{1}{2 \epsilon} \int_{\mathbb{T}^{d}} \chi\left(\frac{f(x)}{\epsilon}\right)\left|\frac{\partial f}{\partial x_{1}}\right| d x=\int_{\vec{y} \in \mathbb{T}^{d-1}}\left(\frac{1}{2 \epsilon} \int_{\left\{t \in \mathbb{T}^{1}:|f(t, \vec{y})| \leq \epsilon\right\}}\left|\frac{\partial f(t, \vec{y})}{\partial t}\right| d t\right) d \vec{y} .
$$

In the inner integral we have for each $\vec{y} \in \mathbb{T}^{d-1}$ a one variable polynomial $g(t)=f(t, \vec{y})$ of degree at most $\sqrt{E}$ and hence by Lemma 3.3, the inner integral is at most $6 \sqrt{E}$. Summing over $j$ introduces another factor of $d$.

As a consequence of the fact that for nonsingular functions we can compute the volume $Z(f)$ of the nodal set of $f \in \mathcal{E}$ via Lemma 3.1 and the fact that almost all $f \in \mathcal{E}$ are nonsingular (Lemma 2.2), we find:

Corollary 3.4. The first and second moments of the volume $Z(f)$ of the nodal set of $f$ are given by

$$
\mathbb{E}(Z)=\mathbb{E}\left(\lim _{\epsilon \rightarrow 0} Z_{\epsilon}\right), \quad \mathbb{E}\left(Z^{2}\right)=\mathbb{E}\left(\lim _{\epsilon_{1}, \epsilon_{2} \rightarrow 0} Z_{\epsilon_{1}} Z_{\epsilon_{2}}\right) .
$$

\section{The EXPeCted Volume of the NOdAL SET}

In this section we show

Proposition 4.1. For $d \geq 1$,

$$
\mathbb{E}(Z)=\mathcal{I}_{d} \sqrt{E}
$$

where

$$
\mathcal{I}_{d}=\sqrt{\frac{4 \pi}{d}} \frac{\Gamma\left(\frac{d+1}{2}\right)}{\Gamma\left(\frac{d}{2}\right)} .
$$

Proof. Since $Z_{\epsilon}$ is uniformly bounded by Lemma 3.2, we can use the Dominated Convergence Theorem to write

$$
\mathbb{E}(Z)=\mathbb{E}\left(\lim _{\epsilon \rightarrow 0} Z_{\epsilon}\right)=\lim _{\epsilon \rightarrow 0} \mathbb{E}\left(Z_{\epsilon}\right)
$$


By Fubini's theorem,

$$
\begin{aligned}
\mathbb{E}\left(Z_{\epsilon}\right)=\mathbb{E}\left(\frac{1}{2 \epsilon} \int_{\mathbb{T}^{d}} \chi\left(\frac{f(x)}{\epsilon}\right)|\nabla f(x)| d x\right) \\
=\int_{\mathbb{T}^{d}} \mathbb{E}\left(\frac{1}{2 \epsilon} \chi\left(\frac{f(x)}{\epsilon}\right)|\nabla f(x)|\right) d x=: \int_{\mathbb{T}^{d}} K_{\epsilon}(x) d x .
\end{aligned}
$$

Now for each $x \in \mathbb{T}^{d}$, the function $f \mapsto \frac{1}{2 \epsilon} \chi\left(\frac{f(x)}{\epsilon}\right)|\nabla f(x)|$ is bounded and hence we may evaluate the integral by using the joint probability density of the variable $(f(x), \nabla f(x))$, whose components are Gaussian of zero mean with covariance

$$
\mathbb{E}\left(f(x)^{2}\right)=1, \quad \mathbb{E}\left(f(x) \frac{\partial f}{\partial x_{j}}(x)\right)=0
$$

and

$$
\mathbb{E}\left(\frac{\partial f(x)}{\partial x_{j}} \frac{\partial f(x)}{\partial x_{k}}\right)=\frac{2}{\mathcal{N}} \cdot 4 \pi^{2} \sum_{\vec{\lambda} \in \Lambda / \pm} \lambda_{j} \lambda_{k}=4 \pi^{2} \frac{E}{d} \cdot \delta_{j, k} .
$$

by (2.2). Thus

$$
\begin{aligned}
K_{\epsilon}(x) & =\frac{1}{2 \epsilon} \int_{\mathbb{R}} \chi\left(\frac{a}{\epsilon}\right) e^{-a^{2} / 2} \frac{d a}{\sqrt{2 \pi}} \int_{\mathbb{R}^{d}}|\vec{b}| \exp \left(-\frac{d|\vec{b}|^{2}}{8 \pi^{2} E}\right) \frac{d \vec{b}}{(2 \pi)^{d / 2}\left(4 \pi^{2} E / d\right)^{d / 2}} \\
& =\frac{\sqrt{4 \pi^{2} E}}{\sqrt{d} \cdot(2 \pi)^{(d+1) / 2}} \int_{\mathbb{R}^{d}}|\vec{v}| \exp \left(-\frac{1}{2}|\vec{v}|^{2}\right) d \vec{v} \frac{1}{2 \epsilon} \int_{\mathbb{R}} \chi\left(\frac{a}{\epsilon}\right) e^{-a^{2} / 2} d a .
\end{aligned}
$$

Integrating over $\mathbb{T}^{d}$ and taking the limit $\epsilon \rightarrow 0$ gives

$$
\mathbb{E}(Z)=\mathcal{I}_{d} \sqrt{E}
$$

where

$$
\mathcal{I}_{d}=\frac{1}{\sqrt{d}(2 \pi)^{(d-1) / 2}} \int_{\mathbb{R}^{d}}|\vec{v}| \exp \left(-\frac{1}{2}|\vec{v}|^{2}\right) d \vec{v} .
$$

In the one-dimensional case, $\mathcal{I}_{1}=\int_{\mathbb{R}}|v| e^{-v^{2} / 2} d v=2$. For $d \geq 2$,

$$
\int_{\mathbb{R}^{d}}|\vec{v}| \exp \left(-\frac{1}{2}|\vec{v}|^{2}\right) d \vec{v}=\operatorname{vol}\left(S^{d-1}\right) \int_{0}^{\infty} r e^{-r^{2} / 2} r^{d-1} d r .
$$

Using

$$
\operatorname{vol}\left(S^{d-1}\right)=\frac{2 \pi^{\frac{d}{2}}}{\Gamma\left(\frac{d}{2}\right)}, \quad \int_{0}^{\infty} r^{d} e^{-r^{2} / 2} d r=2^{\frac{d-1}{2}} \Gamma\left(\frac{d+1}{2}\right)
$$

gives

$$
\int_{\mathbb{R}^{d}}|\vec{v}| \exp \left(-\frac{1}{2}|\overrightarrow{\mid}|^{2}\right) d \vec{v}=\sqrt{2}(2 \pi)^{d / 2} \frac{\Gamma\left(\frac{d+1}{2}\right)}{\Gamma\left(\frac{d}{2}\right)}
$$

(which is consistent with the computation for $d=1$ ). Thus

$$
\mathcal{I}_{d}=\sqrt{\frac{4 \pi}{d}} \frac{\Gamma\left(\frac{d+1}{2}\right)}{\Gamma\left(\frac{d}{2}\right)}
$$

as claimed. 
5. An Integral FORMUla FOR THE SECOND MOMENT

5.1. The covariance matrix. The covariance matrix $\Sigma(x, y)$ of the Gaussian vector $(f(x), f(y), \nabla f(x), \nabla f(y))$ is given by

$$
\Sigma=\left(\begin{array}{cc}
A & B \\
B^{T} & C
\end{array}\right)
$$

where

$$
A=\left(\begin{array}{cc}
\mathbb{E}\left(f(x)^{2}\right) & \mathbb{E}(f(x) f(y)) \\
\mathbb{E}(f(y) f(x)) & \mathbb{E}\left(f(y)^{2}\right)
\end{array}\right), B=\left(\begin{array}{cc}
\mathbb{E}(f(x) \nabla f(x)) & \mathbb{E}(f(x) \nabla f(y)) \\
\mathbb{E}(f(y) \nabla f(x) & \mathbb{E}(f(y) \nabla f(y))
\end{array}\right)
$$

and

$$
C=\left(\begin{array}{ll}
\mathbb{E}\left(\nabla f(x)^{T} \nabla f(x)\right) & \mathbb{E}\left(\nabla f(x)^{T} \nabla f(y)\right) \\
\mathbb{E}\left(\nabla f(y)^{T} \nabla f(x)\right) & \mathbb{E}\left(\nabla f(y)^{T} \nabla f(y)\right)
\end{array}\right) .
$$

For generic $(x, y)$, the covariance matrix $\Sigma(x, y)$ is nonsingular (see Appendix (A).

Lemma 5.1. The covariance matrix $\Sigma(x, y)$ depends only on the difference $z=x-y$ and is given in terms of the two-point function $u$ by

$$
\Sigma(x, y)=\left(\begin{array}{cc}
A(z) & B(z) \\
B(z)^{T} & C(z)
\end{array}\right)
$$

where

$$
A(z)=\left(\begin{array}{cc}
1 & u(z) \\
u(z) & 1
\end{array}\right), \quad B(z)=\left(\begin{array}{cc}
\overrightarrow{0} & -\nabla u(z) \\
\nabla u(z) & \overrightarrow{0}
\end{array}\right)
$$

(here $\overrightarrow{0}, \nabla u$ are row vectors), and

$$
C(z)=\left(\begin{array}{cc}
\frac{4 \pi^{2} E}{d} I & -H(z) \\
-H(z) & \frac{4 \pi^{2} E}{d} I
\end{array}\right)
$$

where $H=\left(\frac{\partial^{2} u}{\partial x_{j} \partial x_{k}}\right)$ is the Hessian of $u$.

Proof. By definition of the two point function, we have $A=\left(\begin{array}{ll}1 & u \\ u & 1\end{array}\right)$. To compute $B$, use

$$
\mathbb{E}\left(\frac{\partial f}{\partial x_{j}}(x) f(y)\right)=\frac{\partial}{\partial x_{j}} \mathbb{E}(f(x) f(y))=\partial_{j} u(x-y)
$$

and hence

$$
\mathbb{E}\left(f(x) \partial_{j} f(y)\right)=\partial_{j} u(y-x)=-\partial_{j} u(x-y) .
$$

In particular

$$
\mathbb{E}(f(x) \nabla f(x))=\overrightarrow{0}
$$

Therefore

$$
B(z)=\left(\begin{array}{cc}
\overrightarrow{0} & -\nabla u(z) \\
\nabla u(z) & \overrightarrow{0}
\end{array}\right)
$$

(where $\overrightarrow{0}$ denotes the $d$-dimensional zero row vector).

To compute $C$, use (4.1) to find

$$
\mathbb{E}\left(\nabla f(x)^{T} \nabla f(x)\right)=\frac{4 \pi^{2} E}{d} I_{d} .
$$


More generally

$$
\mathbb{E}\left(\partial_{j} f(x) \partial_{k} f(y)\right)=\frac{\partial^{2}}{\partial x_{j} \partial y_{k}} \mathbb{E}(f(x) f(y))=-\frac{\partial^{2} u}{\partial x_{j} \partial x_{k}}(x-y)
$$

and so

$$
\mathbb{E}\left(\nabla f(x)^{T} \nabla f(y)\right)=-\left(\frac{\partial^{2} u}{\partial x_{j} \partial x_{k}}(x-y)\right)_{j, k}=-H(x-y) .
$$

Thus

$$
C=\left(\begin{array}{cc}
\frac{4 \pi^{2} E}{d} I & -H \\
-H & \frac{4 \pi^{2} E}{d} I
\end{array}\right)
$$

as claimed.

The inverse of $\Sigma$ (when it exists) is given by

$$
\Sigma^{-1}=\left(\begin{array}{cc}
* & * \\
* & \Omega^{-1}
\end{array}\right)
$$

with $\Omega$ being the $2 d \times 2 d$ matrix

$$
\Omega=C-B^{T} A^{-1} B .
$$

We will call $\Omega$ the reduced covariance matrix. We have

$$
\operatorname{det} \Sigma=\operatorname{det} A \operatorname{det} \Omega=\left(1-u^{2}\right) \operatorname{det} \Omega \text {. }
$$

By Lemma 5.1, we have

$$
\Omega=\left(\begin{array}{cc}
4 \pi^{2}(E / d) I & -H \\
-H & 4 \pi^{2}(E / d) I
\end{array}\right)-\frac{1}{1-u^{2}}\left(\begin{array}{cc}
D^{T} D & u D^{T} D \\
u D^{T} D & D^{T} D
\end{array}\right)
$$

where $D(z)=\nabla u(z)$ and $H=\left(\frac{\partial^{2} u}{\partial x_{j} x_{k}}\right)$ is the Hessian of $u$.

\subsection{A formula for the second moment.}

Proposition 5.2. The second moment of $Z(f)$ is given by

$$
\mathbb{E}\left(Z^{2}\right)=\int_{\mathbb{T}^{d}} K(x) d x
$$

where

$$
K(x)=\frac{1}{\sqrt{1-u^{2}}} \int_{\mathbb{R}^{2 d}}\left\|v_{1}\right\|\left\|v_{2}\right\| \frac{\exp \left(-\frac{1}{2} v \Omega^{-1} v^{T}\right)}{\sqrt{\operatorname{det} \Omega}} \frac{d v}{(2 \pi)^{d+1}} .
$$

Denote

$$
K_{\epsilon_{1}, \epsilon_{2}}(x, y):=\frac{1}{4 \epsilon_{1} \epsilon_{2}} \int_{\mathcal{E}}\|\nabla f(x)\|\|\nabla f(y)\| \chi\left(\frac{f(x)}{\epsilon_{1}}\right) \chi\left(\frac{f(y)}{\epsilon_{2}}\right) d \mu(f) .
$$

We have the following

Lemma 5.3. For $(x, y) \in \mathbb{T}^{d} \times \mathbb{T}^{d}$ with $u(x-y)^{2} \neq 1$

$$
K_{\epsilon_{1}, \epsilon_{2}}(x, y) \ll_{d} \frac{E}{\sqrt{1-u^{2}(x-y)}},
$$

where the implied constant depends only on the dimension $d$. 
Proof. Write $f(x)=\langle f, U(x)\rangle$, where $U(x)$ is the unit vector

$$
U(x)=\frac{\sqrt{2}}{\sqrt{\mathcal{N}}}(\cos 2 \pi\langle\lambda, x\rangle, \sin 2 \pi\langle\lambda, x\rangle)_{\lambda \in \Lambda / \pm} \in S^{\mathcal{N}-1},
$$

and where we identify the function $f$ with a vector in $\mathbb{R}^{\mathcal{N}}$ via (2.1). Note that $\langle U(x), U(y)\rangle=u(x-y)$ is the cosine of the angle between $U(x)$ and $U(y)$.

We have

$$
\nabla f(x)=D U \cdot f
$$

where the derivative $D U$ is a $d \times \mathcal{N}$ matrix. Equivalently,

$$
(\nabla f(x))_{i}=\left\langle f,\left(\frac{\partial}{\partial x_{i}} U(x)\right)\right\rangle, \quad 1 \leq i \leq d .
$$

By the triangle and Cauchy-Schwartz inequalities,

$$
\|\nabla f(x)\| \leq \sum_{i=1}^{d}\|f\| \cdot\left\|\left(\frac{\partial}{\partial x_{i}} U(x)\right)\right\| \ll \sqrt{E}\|f\|,
$$

by a computation of $\frac{\partial U}{\partial x_{i}}$. Therefore

$$
K_{\epsilon_{1}, \epsilon_{2}}(x, y) \ll \frac{E}{4 \epsilon_{1} \epsilon_{2}} \int_{\substack{|f(x)|<\epsilon_{1} \\|f(y)|<\epsilon_{2}}}\|f\|^{2} e^{-\|f\|^{2} / 2} d f .
$$

Consider the plane $\pi \subset \mathbb{R}^{\mathcal{N}}$ spanned by $U(x)$ and $U(y)$. The domain of the integration is all the vectors $f \in \mathbb{R}^{\mathcal{N}}$ so that the projection of $f$ on $\pi$ falls into the parallelogram $P$ of lengths $2 \epsilon_{1}$ and $2 \epsilon_{2}$. The cosine of the angle between the sides of $P$ is $\langle U(x), U(y)\rangle=u(x-y)$. Therefore the area of $P$ is

$$
\operatorname{area}(P)=4 \epsilon_{1} \epsilon_{2} \frac{1}{\sqrt{1-u(x-y)^{2}}} .
$$

Write the multiple integral in (5.7) as the iterated integral

$$
\int_{P}\left(\int_{p+\pi^{\perp}}\|f\|^{2} e^{-\|f\|^{2} / 2} d f\right) d p
$$

where the variable $p$ runs over all the points of the parallelepiped $P$. The inner integral in (5.8) is $O(1)$ with the constant depending on $d$ only. Indeed, note that for every $f_{1} \in \pi^{\perp}$,

$$
\begin{aligned}
\left\|p+f_{1}\right\|^{2} e^{-\left\|p+f_{1}\right\|^{2} / 2} & =\left(\|p\|^{2}+\left\|f_{1}\right\|^{2}\right) e^{-\left(\|p\|^{2}+\left\|f_{1}\right\|^{2}\right) / 2} \\
& \ll\left(1+\left\|f_{1}\right\|^{2}\right) \cdot e^{-\left\|f_{1}\right\|^{2} / 2},
\end{aligned}
$$

since $\|p\|^{2} e^{-\|p\|^{2} / 2}$ is bounded. Our claim follows from convergence of the integral $\int_{\mathbb{R}^{\mathcal{N}-2}}\left(1+\|w\|^{2}\right) e^{-\|w\|^{2} / 2} d w$. Therefore

$$
\int_{\substack{|f(x)|<\epsilon_{1} \\|f(y)|<\epsilon_{2}}}\|f\|^{2} e^{-\|f\|^{2} / 2} d f \ll \operatorname{area}(P) \ll \epsilon_{1} \epsilon_{2} \frac{1}{\sqrt{1-u(x-y)^{2}}} .
$$

Substituting the last estimate into (5.7) proves (5.6). 
Proof of proposition 5.2. By Corollary 3.4, we have

$$
\begin{aligned}
& \mathbb{E}\left(Z^{2}\right)= \\
& \int_{\mathcal{E}}\left[\lim _{\epsilon_{1}, \epsilon_{2} \rightarrow 0} \frac{1}{2 \epsilon_{1}} \int_{\mathbb{T}^{d}}\|\nabla f(x)\| \chi\left(\frac{f(x)}{\epsilon_{1}}\right) d x \frac{1}{2 \epsilon_{2}} \int_{\mathbb{T}^{d}}\|\nabla f(y)\| \chi\left(\frac{f(y)}{\epsilon_{2}}\right) d y\right] d \mu(f)
\end{aligned}
$$

where $\mu$ is the Gaussian measure $d \mu(f)=e^{-\|f\|^{2} / 2} \frac{d f}{(2 \pi)^{\mathcal{N} / 2}}$. We wish to change the order of the limit and the integration. To do so, we notice that by Lemma 3.2, the integrand is bounded by $O(E)$. Therefore, the change of order follows from the dominated convergence theorem. Thus the integral equals

$$
\lim _{\epsilon_{1}, \epsilon_{2} \rightarrow 0} \frac{1}{4 \epsilon_{1} \epsilon_{2}} \int_{\mathcal{E}} \int_{\left(\mathbb{T}^{d}\right)^{2}}\|\nabla f(x)\|\|\nabla f(y)\| \chi\left(\frac{f(x)}{\epsilon_{1}}\right) \chi\left(\frac{f(y)}{\epsilon_{2}}\right) d x d y d \mu(f) .
$$

Using Fubini's theorem, this equals to

$$
\lim _{\epsilon_{1}, \epsilon_{2} \rightarrow 0} \frac{1}{4 \epsilon_{1} \epsilon_{2}} \int_{\left(\mathbb{T}^{d}\right)^{2}} \int_{\mathcal{E}}\|\nabla f(x)\|\|\nabla f(y)\| \chi\left(\frac{f(x)}{\epsilon_{1}}\right) \chi\left(\frac{f(y)}{\epsilon_{2}}\right) d \mu(f) d x d y .
$$

Now we wish to exchange the order of taking limit and the integration over $\left(\mathbb{T}^{d}\right)^{2}$. To justify it, we use the dominated convergence theorem with Lemma 5.3. The upper bound for $u(x) \neq \pm u(y)$ is sufficient, since this happens for almost all $(x, y) \in\left(\mathbb{T}^{d}\right)^{2}$, and changing the values of a function on a set of measure 0 does not have any impact on the integrability and the value of the integral of a function. The convergence of the RHS of (5.6) was shown in 11]. Therefore, we may exchange the order of the limit and the integral in (5.9) to obtain

$$
\mathbb{E}\left(Z^{2}\right)=\int_{\mathbb{T}^{d} \times \mathbb{T}^{d}} K(x, y) d x d y
$$

where

$$
K(x, y)=\lim _{\epsilon_{1}, \epsilon_{2} \rightarrow 0} K_{\epsilon_{1}, \epsilon_{2}}(x, y) .
$$

We will replace the vector $(f(x), f(y), \nabla f(x), \nabla f(y))$ with a $2 d+2$ dimensional Gaussian vector with covariance matrix $\Sigma(x, y)=\Sigma(x-y)$ defined in (5.1). The proof that for almost all $x, y$ this is indeed a $2 d+2$ dimensional process, is relegated to Proposition A.1 in the Appendix. This gives

$$
\begin{aligned}
& K_{\epsilon_{1}, \epsilon_{2}}(x, y) \\
& =\frac{1}{4 \epsilon_{1} \epsilon_{2}} \int_{\mathbb{R}^{2 d+2}}\left\|v_{1}\right\|\left\|v_{2}\right\| \chi\left(\frac{w_{1}}{\epsilon_{1}}\right) \chi\left(\frac{w_{2}}{\epsilon_{2}}\right) e^{-(v, w) \Sigma^{-1}(v, w)^{T} / 2} \frac{d v d w}{\sqrt{\operatorname{det} \Sigma}(2 \pi)^{d+1}} \\
& =\frac{1}{4 \epsilon_{1} \epsilon_{2}} \int_{-\epsilon_{1}}^{\epsilon_{1}} \int_{-\epsilon_{2}}^{\epsilon_{2}} \int_{\mathbb{R}^{2 d}}\left\|v_{1}\right\|\left\|v_{2}\right\| e^{-(v, w) \Sigma^{-1}(v, w)^{T} / 2} \frac{d v d w}{\sqrt{\operatorname{det} \Sigma}(2 \pi)^{d+1}} .
\end{aligned}
$$

We therefore have

$$
\begin{aligned}
& K(x, y)= \\
& \lim _{\epsilon_{1}, \epsilon_{2} \rightarrow 0} \frac{1}{4 \epsilon_{1} \epsilon_{2}} \int_{-\epsilon_{1}}^{\epsilon_{1}} \int_{-\epsilon_{2}}^{\epsilon_{2}} \int_{\mathbb{R}^{2 d}}\left\|v_{1}\right\|\left\|v_{2}\right\| e^{-(v, w) \Sigma^{-1}(v, w)^{T} / 2} \frac{d v d w}{\sqrt{\operatorname{det} \Sigma}(2 \pi)^{d+1}} .
\end{aligned}
$$


Since the last integrand is continuous, we may use the fundamental theorem of the calculus to replace the averaging over $w_{1}, w_{2}$ by the value at $w_{1}=$ $w_{2}=0$, to obtain

$$
K(x, y)=\int_{\mathbb{R}^{2 d}}\left\|v_{1}\right\|\left\|v_{2}\right\| e^{-(v, \overrightarrow{0}) \Sigma^{-1}(v, \overrightarrow{0})^{T} / 2} \frac{d v d w}{\sqrt{\operatorname{det} \Sigma}(2 \pi)^{d+1}} .
$$

We have

$$
\Sigma^{-1}=\left(\begin{array}{cc}
* & * \\
* & \Omega^{-1}
\end{array}\right)
$$

with $\Omega=C-B^{T} A^{-1} B$ being the reduced covariance matrix, which we computed in (5.3). Together with (5.2) we find

$$
K(x, y)=\frac{1}{\sqrt{1-u^{2}}} \int_{\mathbb{R}^{2 d}}\left|v_{1}\right|\left|v_{2}\right| \frac{\exp \left(-\frac{1}{2} v \Omega^{-1} v^{T}\right)}{\sqrt{\operatorname{det} \Omega}} \frac{d v}{(2 \pi)^{d+1}} .
$$

Finally, we get Proposition 5.2 by noticing that $K(x, y)=K(x-y)$, and therefore the (double) integral in (5.10) may be expressed as a single integral.

In the course of the proof we saw that $K(x, y)=\lim _{\epsilon_{1}, \epsilon_{2} \rightarrow 0} K_{\epsilon_{1}, \epsilon_{2}}(x)$. Therefore, taking the limit $\epsilon_{1}, \epsilon_{2} \rightarrow 0$ and using Lemma 5.3 we obtain

Corollary 5.4. If $u(x)^{2} \neq 1$ then

$$
K(x) \ll \frac{E}{\sqrt{1-u(x)^{2}}} .
$$

\section{A BOUND FOR THE VARIANCE}

In this section we prove:

Theorem 6.1. For $d \geq 2$,

$$
\operatorname{Var}(Z)=O\left(\frac{E}{\sqrt{\mathcal{N}}}\right) .
$$

6.1. Plan of the proof. We use the integral formula (5.4) for the second moment of $Z(f)$, that is $\mathbb{E}\left(Z^{2}\right)=\int_{\mathbb{T}^{d}} K(z) d z$, with

$$
K(z)=\frac{1}{\sqrt{1-u^{2}}} \int_{\mathbb{R}^{2 d}}\left\|v_{1}\right\|\left\|v_{2}\right\| \frac{\exp \left(-\frac{1}{2} v \Omega^{-1} v^{T}\right)}{\sqrt{\operatorname{det} \Omega}} \frac{d v}{(2 \pi)^{d+1}} .
$$

As in [11, we will define a notion of "singular points" in $\mathbb{T}^{d}$ where the factor $1 / \sqrt{1-u^{2}}$ is large, and treat separately the singular and nonsingular points. The singular set is shown to give a contribution of $O(E / \mathcal{N})$. On the nonsingular set, the factor $1 / \sqrt{1-u^{2}}$ may, up to an admissible error, safely be replaced by 1 . To treat the Gaussian integral, we write

$$
\Omega(z)=\frac{4 \pi^{2} E}{d}(I-S(z))
$$

and recover the square of the expected value $\mathbb{E}(Z)^{2}$ from the contribution of the identity matrix $I$; the rest is then the key quantity for bounding the variance. Setting $\sigma(z)$ to be the spectral norm of $S(z)$, we show that that variance is bounded by $E\left(\int_{\mathbb{T}^{d}} \sigma(z) d z+O(1 / \mathcal{N})\right)$. Now $\sigma(z)$ is at most 
$\sqrt{\operatorname{tr}\left(S(z)^{2}\right)}$, whose integral we need to bound. We do this by using CauchySchwartz, which allows us to bound it by $\left(\int_{\mathbb{T}^{d}} \operatorname{tr}\left(S(z)^{2}\right) d z\right)^{1 / 2} \ll 1 / \sqrt{\mathcal{N}}$. Hence the variance is $\operatorname{Var}(Z) \ll E / \sqrt{\mathcal{N}}$. It should be possible to improve this to $O(E / \mathcal{N})$.

6.2. The singular set. We give the definition of [11] for singular points:

Definition 6.2. A point $x \in \mathbb{T}^{d}$ is a positive singular point if there is a set of frequencies $\Lambda_{x} \subset \Lambda$ with density $\frac{\left|\Lambda_{x}\right|}{|\Lambda|}>1-\frac{1}{4 d}$ for which $\cos 2 \pi\langle\lambda, x\rangle>3 / 4$ for all $\lambda \in \Lambda_{x}$. Similarly we define a negative singular point to be a point $x$ where there is a set $\tilde{\Lambda}_{x} \subset \Lambda$ of density $>1-\frac{1}{4 d}$ for which $\cos 2 \pi\langle\lambda, x\rangle<-3 / 4$ for all $\lambda \in \tilde{\Lambda}_{x}$.

Let $M \approx \sqrt{E}$ be a large integer. We decompose the torus $\mathbb{T}^{d}=\mathbb{R}^{d} / \mathbb{Z}^{d}$ as a disjoint union (with boundary overlaps) of $M^{d}$ closed cubes $I_{\vec{k}}$ of side length $1 / M$ centered at $\vec{k} / M, \vec{k} \in \mathbb{Z}^{d}$.

Definition 6.3. A cube $I_{\vec{k}}$ is a positive (resp. negative) singular cube if it contains a positive (resp. negative) singular point.

Definition 6.4. The singular set $B$ is the union of all singular cubes.

In [11], we showed that the measure of the singular set is bounded by

$$
\operatorname{meas}(B) \ll \int_{\mathbb{T}^{d}} u(x)^{4} d x \ll \frac{1}{\mathcal{N}}
$$

(and except in dimensions $d=3,4$ this is $o(1 / \mathcal{N})$ ).

In order to bound the contribution of the singular set to the integral in (5.4), we use Corollary [5.4. It was shown in [11] (see (6.3)) that

$$
\int_{B} \frac{d x}{\sqrt{1-u(x)^{2}}} \ll \int_{\mathbb{T}^{d}} u(x)^{4} d x \ll \frac{1}{\mathcal{N}} .
$$

Therefore we obtain:

Corollary 6.5. The contribution of the singular set is bounded by

$$
\int_{B} K(x) d x \ll E \int_{\mathbb{T}^{d}} u(x)^{4} d x \ll \frac{E}{\mathcal{N}} .
$$

6.3. The nonsingular set. We now want to estimate the contribution of the nonsingular set to the integral formula of Proposition 5.2 for the second moment of $Z$. Recall that it reads $\mathbb{E}\left(Z^{2}\right)=\int_{\mathbb{T}^{d}} K(z) d z$ with the kernel $K(z)$ given by (5.5), that is

$$
K(z)=\frac{1}{\sqrt{1-u(z)^{2}}} \int_{\mathbb{R}^{2 d}}\left\|v_{1}\right\|\left\|v_{2}\right\| \frac{\exp \left(-\frac{1}{2} v \Omega(z)^{-1} v^{T}\right)}{\sqrt{\operatorname{det} \Omega(z)}} \frac{d v}{(2 \pi)^{d+1}} .
$$

A consequence of the definition of singular points is that on the nonsingular set, $u$ is bounded away from \pm 1 . In [11, lemma 6.5] we showed that if $x \in \mathbb{T}^{d}$ is nonsingular then

$$
|u(x)|<1-\frac{1}{16 d} .
$$


As a consequence, on the nonsingular set, we may expand

$$
\frac{1}{\sqrt{1-u^{2}}}=1+O\left(u^{2}\right)
$$

where the implied constant depends only on $d$.

We now wish to handle the "reduced covariance matrix" $\Omega$ of (5.3) on the nonsingular set. We write $\Omega=\left(4 \pi^{2} E / d\right) \cdot \Omega_{1}$ and $\Omega_{1}=I-S$, where

$$
S=\frac{d}{4 \pi^{2} E} \frac{1}{1-u^{2}}\left(\begin{array}{cc}
D^{T} D & \left(1-u^{2}\right) H+u D^{T} D \\
\left(1-u^{2}\right) H+u D^{T} D & D^{T} D
\end{array}\right)
$$

Note that since outside a set of measure zero, $\Omega_{1} \gg 0$ is positive definite, we have $S \ll I$ in the sense that all eigenvalues of $S$ are in $(-\infty, 1)$. Let $\sigma$ be the spectral norm of $S$, so that denoting the eigenvalues of $S$ by $\alpha_{1}, \ldots, \alpha_{2 d}$,

$$
\sigma=\max _{1 \leq j \leq 2 d}\left|\alpha_{j}\right| .
$$

We give a bound on the mean and the mean-square of $\sigma$ on the complement $B^{c}$ of the singular set.

\section{Lemma 6.6.}

$$
\int_{B^{c}} \sigma^{2} d x \ll \frac{1}{\mathcal{N}}
$$

and

$$
\int_{B^{c}} \sigma d x \ll \frac{1}{\sqrt{\mathcal{N}}} .
$$

Proof. The bound (6.5) follows from (6.4) by applying Cauchy-Schwartz, so it suffices to prove (6.4). We have $\sigma^{2} \leq \sum \alpha_{j}^{2}=\operatorname{tr}\left(S^{2}\right)$, and so it suffices to show

$$
\int_{B^{c}} \operatorname{tr}\left(S^{2}\right) d x \ll \frac{1}{\mathcal{N}} .
$$

On the nonsingular set, the expression $\frac{1}{1-u^{2}}$ is bounded, and hence for purposes of upper bounds may be ignored. The entries of $S^{2}$ on the nonsingular set are thus bounded by sums of the following expressions :

$$
\frac{1}{E^{2}} \frac{\partial^{2} u}{\partial x_{i} \partial x_{j}} \frac{\partial^{2} u}{\partial x_{k} \partial x_{\ell}}, \quad \frac{1}{E^{2}} \frac{\partial^{2} u}{\partial x_{i} \partial x_{j}} \frac{\partial u}{\partial x_{k}} \frac{\partial u}{\partial x_{\ell}}, \quad \frac{1}{E^{2}} \frac{\partial u}{\partial x_{i}} \frac{\partial u}{\partial x_{j}} \frac{\partial u}{\partial x_{k}} \frac{\partial u}{\partial x_{\ell}}
$$

and it suffices to show that the integral of each over all of $\mathbb{T}^{d}$ is $O(1 / \mathcal{N})$.

By applying Cauchy-Schwartz, it suffices to show

$$
\int_{\mathbb{T}^{d}}\left(\frac{\partial^{2} u}{\partial x_{i} \partial x_{j}}\right)^{2} d x \ll \frac{E^{2}}{\mathcal{N}} \quad \text { and } \quad \int_{\mathbb{T}^{d}}\left(\frac{\partial u}{\partial x_{k}}\right)^{4} d x \ll \frac{E^{2}}{\mathcal{N}} .
$$

We have

$$
\frac{\partial^{2} u}{\partial x_{i} \partial x_{j}}=\frac{-8 \pi^{2}}{\mathcal{N}} \sum_{\lambda \in \Lambda / \pm} \lambda_{j} \lambda_{k} \cos 2 \pi\langle\lambda, x\rangle
$$


and hence

$$
\begin{aligned}
\int_{\mathbb{T}^{d}}\left(\frac{\partial^{2} u}{\partial x_{i} \partial x_{j}}\right)^{2} d x & =\left(\frac{8 \pi^{2}}{\mathcal{N}}\right)^{2} \sum_{\lambda, \mu \in \Lambda / \pm} \lambda_{i} \lambda_{j} \mu_{i} \mu_{j} \frac{1}{2} \delta(\lambda, \mu) \\
& \ll \frac{1}{\mathcal{N}^{2}} \sum_{\lambda \in \Lambda} \lambda_{i}^{2} \lambda_{j}^{2} \ll \frac{E^{2}}{\mathcal{N}}
\end{aligned}
$$

since $\lambda_{j}^{2} \leq|\lambda|^{2}=E$.

To bound $\int_{\mathbb{T}^{d}}\left(\frac{\partial r}{\partial x_{k}}\right)^{4} d x$, we write

$$
\frac{\partial u}{\partial x_{k}}=\frac{-4 \pi}{\mathcal{N}} \sum_{\lambda \in \Lambda / \pm} \lambda_{k} \sin 2 \pi\langle\lambda, x\rangle
$$

and as above, we have

$$
\int_{\mathbb{T}^{d}}\left(\frac{\partial u}{\partial x_{k}}\right)^{4} d x \ll \frac{1}{\mathcal{N}^{4}} \sum_{\substack{\lambda^{1}, \ldots, \lambda^{4} \in \Lambda / \pm \lambda^{1} \pm \lambda^{2} \pm \lambda^{3} \pm \lambda^{4}=0}} \lambda_{k}^{1} \cdot \lambda_{k}^{2} \cdot \lambda_{k}^{3} \cdot \lambda_{k}^{4} \ll \frac{E^{2}}{\mathcal{N}}
$$

since $\lambda^{1}, \lambda^{2}, \lambda^{3}$ determine $\lambda^{4}$ once we decree that $\lambda^{1} \pm \lambda^{2} \pm \lambda^{3} \pm \lambda^{4}=0$, and $\left|\lambda_{k}^{i}\right| \ll \sqrt{E}$.

6.4. Concluding the proof of Theorem 6.1. Since $\Omega_{1}=\Omega \cdot d /\left(4 \pi^{2} E\right)$ is symmetric and positive definite (away from a set of measure zero), it has a positive definite square root $P_{1}=P_{1}^{T} \gg 0, \Omega_{1}=P_{1}^{2}$. By proposition 5.2

$$
\begin{aligned}
K(x) & =\frac{1}{\sqrt{1-u^{2}}} \int_{\mathbb{R}^{2 d}}\left|\vec{v}_{1}\right|\left|\vec{v}_{2}\right| \frac{\exp \left(-\frac{1}{2} \vec{v} \Omega^{-1} \vec{v}^{T}\right)}{\sqrt{\operatorname{det} \Omega}} \frac{d \vec{v}}{(2 \pi)^{d+1}} \\
& \left.=\frac{4 \pi^{2} E}{d} \frac{1}{\sqrt{1-u^{2}}} \int_{\mathbb{R}^{2 d}} \mid \overrightarrow{(z} P_{1}\right)_{1}||\left(\vec{z} P_{1}\right)_{2} \mid e^{-|\vec{z}|^{2} / 2} \frac{d \vec{z}}{(2 \pi)^{d+1}}
\end{aligned}
$$

on using the change of variables $\vec{v}=\frac{2 \pi \sqrt{E}}{\sqrt{d}} \cdot \vec{z} \cdot P_{1}$.

We claim that

$$
P_{1}=I(1+O(\sigma)) .
$$

Indeed, if $S=U D U^{T}, U$ orthogonal and $D=\operatorname{diag}\left(\alpha_{1}, \ldots, \alpha_{2 d}\right)$ then $P_{1}=$ $U(I-D)^{1 / 2} U^{T}$ and using the inequality $|\sqrt{1-\alpha}-1|<|\alpha|$ for $-\infty<\alpha<1$, gives

$$
(I-D)^{1 / 2}=I+O\left(\left(\begin{array}{ccc}
\left|\alpha_{1}\right| & & \\
& \ddots & \\
& & \left|\alpha_{2 d}\right|
\end{array}\right)\right)=I(1+O(\sigma))
$$

Thus we may write $z P_{1}=z(1+O(\sigma))$.

On the nonsingular set, we may expand

$$
\frac{1}{\sqrt{1-u^{2}}}=1+O\left(u^{2}\right)
$$


and so we find that on the nonsingular set

$$
\begin{aligned}
K(x) & =\frac{4 \pi^{2} E}{d} \int_{\mathbb{R}^{2 d}}\left|\vec{z}_{1} \| \vec{z}_{2}\right| e^{-|\vec{z}|^{2} / 2}\left(1+O\left(u^{2}\right)\right)(1+O(\sigma))^{2} \frac{d \vec{z}}{(2 \pi)^{d+1}} \\
& =\mathbb{E}(Z)^{2}\left(1+O\left(u^{2}\right)+O(\sigma)+O\left(\sigma^{2}\right)\right) .
\end{aligned}
$$

Integrating over the nonsingular set, and using

$$
\int_{B^{c}} 1=1+O(\operatorname{meas}(B))
$$

we find

$$
\frac{\int_{B^{c}} K(x) d x}{\mathbb{E}\left(Z^{2}\right)}=1+O\left(\int_{B^{c}}\left(u^{2}+\sigma+\sigma^{2}\right) d x\right)+O(\operatorname{meas}(B)) .
$$

Now $\int_{\mathbb{T}^{d}} u(x)^{2} d x=\frac{1}{\mathcal{N}}$, and by lemma 6.6

$$
\int_{B^{c}} \sigma^{2} d x \ll \frac{1}{\mathcal{N}}, \quad \int_{B^{c}} \sigma d x \ll \frac{1}{\sqrt{\mathcal{N}}} .
$$

Furthermore, by (6.1),

$$
\operatorname{meas}(B) \ll \int_{\mathbb{T}^{d}} u^{4} d x \ll \frac{1}{\mathcal{N}},
$$

so that we find

$$
\int_{B^{c}} K(x) d x=\mathbb{E}\left(Z^{2}\right)\left(1+O\left(\frac{1}{\sqrt{\mathcal{N}}}\right)\right) .
$$

By Corollary 6.5, the singular set contributes at most

$$
\int_{B} K(x) d x \ll \frac{E}{\mathcal{N}} .
$$

Therefore we find

$$
\mathbb{E}\left(Z^{2}\right)=\mathbb{E}(Z)^{2}+O\left(\frac{E}{\sqrt{\mathcal{N}}}\right)
$$

that is

$$
\operatorname{Var}(Z) \ll \frac{E}{\sqrt{\mathcal{N}}} .
$$

Thus we have concluded the proof of Theorem 6.1.

Appendix A. The non-Degeneracy of the Covariance matrix

In this appendix we show that the covariance matrix defined by (4.1) is nonsingular for almost all $(x, y) \in\left(\mathbb{T}^{d}\right)^{2}$, thereby justifying the change of variables (5.11).

Proposition A.1. Assume that $\mathcal{N} \gg_{d} 1$ and $d \geq 2$. Then for almost all $(x, y) \in \mathbb{T}^{d} \times \mathbb{T}^{d}$ the linear map $\mathcal{E} \rightarrow \mathbb{R}^{2 d+2}$ defined by

$$
f \mapsto(f(x), f(y), \nabla f(x), \nabla f(y)),
$$

is surjective. 
We want to show that for almost all pairs $(x, y) \in \mathbb{T}^{d} \times \mathbb{T}^{d}$, the only vector $(\alpha, \beta, \vec{C}, \vec{D}) \in \mathbb{R}^{2 d+2}$ satisfying

$$
\alpha f(x)+\beta f(y)+\frac{1}{2 \pi}\langle C, \nabla f(x)\rangle+\frac{1}{2 \pi}\langle D, \nabla f(y)\rangle=0, \quad \forall f \in \mathcal{E}
$$

is the zero vector. Taking $f(x)=e^{2 \pi i\langle\lambda, x\rangle}, \lambda \in \Lambda$ gives

$$
\alpha e^{2 \pi i\langle\lambda, x\rangle}+\beta e^{2 \pi i\langle\lambda, y\rangle}+i e^{2 \pi i\langle\lambda, x\rangle}\langle C, \lambda\rangle+i e^{2 \pi i\langle\lambda, y\rangle}\langle D, \lambda\rangle=0, \quad \forall \lambda \in \Lambda
$$

or setting $z=y-x$,

$$
\alpha+i\langle C, \lambda\rangle=-e^{2 \pi i\langle\lambda, z\rangle}(\beta+i\langle D, \lambda\rangle), \quad \forall \lambda \in \Lambda .
$$

Thus we are reduced to proving the following:

Lemma A.2. Assume that $\mathcal{N} \gg_{d} 1$ and $d \geq 2$. Then for almost all $z$, the only solution for the equation

$$
\alpha+i\langle C, \lambda\rangle=-e^{2 \pi i\langle\lambda, z\rangle}(\beta+i\langle D, \lambda\rangle), \quad \forall \lambda \in \Lambda,
$$

is $\alpha=\beta=0, C=D=\overrightarrow{0}$.

Proof. We divide the work into two steps: In the first step, we show that for all $z \in \mathbb{T}^{d}$, the solutions of (A.1) satisfy $\beta= \pm \alpha$ and $D= \pm C$. In the second step, we take $\beta= \pm \alpha$ and $D= \pm C$ and show that for almost all $z \in \mathbb{T}^{d}$, the only solutions of (A.1) are $\alpha=0$ and $C=\overrightarrow{0}$.

Step 1: We first show that for all $z \in \mathbb{T}^{d}$, all solutions of (A.1) satisfy $\beta= \pm \alpha$ and $D= \pm C$.

Taking squared norms of both sides of (A.1), we get

$$
\alpha^{2}+\langle C, \lambda\rangle^{2}=\beta^{2}+\langle D, \lambda\rangle^{2}, \quad \forall \lambda \in \Lambda
$$

or

$$
\langle C, \lambda\rangle^{2}-\langle D, \lambda\rangle^{2}=\beta^{2}-\alpha^{2}, \quad \forall \lambda \in \Lambda .
$$

Setting $A=C-D=\left(a_{1}, a_{2}, \ldots\right)$ and $B=C+D=\left(b_{1}, b_{2}, \ldots\right)$, we have

$$
\langle A, \lambda\rangle \cdot\langle B, \lambda\rangle=\beta^{2}-\alpha^{2}, \quad \forall \lambda \in \Lambda
$$

and it suffices to see that $A=\overrightarrow{0}$ or $B=\overrightarrow{0}$.

If $\mathcal{N} \gg_{d} 1$, then there is some $\lambda \in \Lambda$ with two nonzero coordinates, say $\lambda_{1} \lambda_{2} \neq 0$ (by applying a permutation of the coordinates to $\lambda$ we may replace 1 and 2 by any pair of distinct indices). For each $\epsilon \in\{ \pm 1\}^{d}$, replace $\lambda$ in (A.2) by

$$
\lambda^{\epsilon}:=\left(\epsilon_{1} \lambda_{1}, \epsilon_{2} \lambda_{2}, \ldots, \epsilon_{d} \lambda_{d}\right)
$$

multiply the result by

$$
\chi_{1,2}(\epsilon)=\epsilon_{1} \epsilon_{2}
$$

and sum the resulting equalities over all $\epsilon \in\{ \pm 1\}^{d}$, using

to get

$$
\sum_{\epsilon \in\{ \pm 1\}^{d}} \chi_{1,2}(\epsilon)=0
$$

$$
\sum_{\epsilon \in\{ \pm 1\}^{d}} \chi_{1,2}(\epsilon)\left\langle A, \lambda^{\epsilon}\right\rangle \cdot\left\langle B, \lambda^{\epsilon}\right\rangle=0
$$


Expanding

$$
\left\langle A, \lambda^{\epsilon}\right\rangle \cdot\left\langle B, \lambda^{\epsilon}\right\rangle=\sum_{j, k=1}^{d} \epsilon_{j} \epsilon_{k} a_{j} b_{k} \lambda_{j} \lambda_{k}
$$

and using

$$
\sum_{\epsilon \in\{ \pm 1\}^{d}} \chi_{1,2}(\epsilon) \epsilon_{j} \epsilon_{k}= \begin{cases}2^{d}, & (j, k)=(1,2) \text { or }(2,1) \\ 0 & \text { otherwise }\end{cases}
$$

we get

$$
2^{d} \lambda_{1} \lambda_{2}\left(a_{1} b_{2}+a_{2} b_{1}\right)=0
$$

and since we assume $\lambda_{1} \lambda_{2} \neq 0$, we find $a_{1} b_{2}+a_{1} b_{2}=0$. Repeating the argument with any pair of distinct indices finally shows that

$$
a_{i} b_{j}+a_{j} b_{i}=0, \quad \forall i \neq j .
$$

If $A \neq \overrightarrow{0}$, say $a_{1} \neq 0$, then we find that

$$
b_{j}=-\frac{b_{1}}{a_{1}} a_{j}, \quad \forall j \neq 1 .
$$

Thus if $b_{1}=0$ then all $b_{j}=0$, that is $B=\overrightarrow{0}$ and we are done. Therefore we may assume that $b_{1} \neq 0$ (and we have also assumed $a_{1} \neq 0$ ). We will show this cannot happen.

If $d>2$, we substitute (A.4) in (A.3) with any $i \neq 1, j \neq 1$ to get

$$
2 \frac{b_{1}}{a_{1}} a_{i} a_{j}=0
$$

that is since $b_{1} \neq 0$, that

$$
a_{i} a_{j}=0, \quad \forall i \neq j, \quad i, j \neq 1 .
$$

Thus there is at most one index $k \neq 1$ with $a_{k} \neq 0$, say $k=2$, so we find that $a_{j}=0$ for $j \neq 1,2$, and by (A.4) we therefore have $b_{j}=0$ for $j \neq 1,2$. Thus

$$
A=\left(a_{1}, a_{2}, \overrightarrow{0}\right), \quad B=\frac{b_{1}}{a_{1}}\left(a_{1},-a_{2}, \overrightarrow{0}\right)
$$

(if $d=2$ this still holds, we just ignore the extra coordinates).

Plugging this into (A.2) with $\lambda$ so that $\lambda_{1} \neq \pm \lambda_{2}$ (which exists if $\mathcal{N} \gg_{d} 1$ ) gives

$$
\left(a_{1} \lambda_{1}\right)^{2}-\left(a_{2} \lambda_{2}\right)^{2}=\frac{a_{1}}{b_{1}}\left(\beta^{2}-\alpha^{2}\right)
$$

and replacing $\lambda=\left(\lambda_{1}, \lambda_{2}, \ldots\right)$ with $\left(\lambda_{2}, \lambda_{1}, \ldots\right)$ gives

$$
\left(a_{1} \lambda_{2}\right)^{2}-\left(a_{2} \lambda_{1}\right)^{2}=\frac{a_{1}}{b_{1}}\left(\beta^{2}-\alpha^{2}\right) .
$$

Comparing (A.5) with (A.6) gives

$$
\left(a_{1} \lambda_{1}\right)^{2}-\left(a_{2} \lambda_{2}\right)^{2}=\left(a_{1} \lambda_{2}\right)^{2}-\left(a_{2} \lambda_{1}\right)^{2}
$$

that is

$$
\left(\lambda_{1}^{2}-\lambda_{2}^{2}\right)\left(a_{1}^{2}+a_{2}^{2}\right)=0 .
$$

Since we chose $\lambda_{2} \neq \pm \lambda_{1}$ this gives $a_{1}=a_{2}=0$, contradicting $a_{1} \neq 0$. Thus we are done with step 1 . 
Step 2: We take $C= \pm D$ and $\alpha= \pm \beta$ in (A.1) and wish to show that for almost all $z \in \mathbb{T}^{d}$, the only solutions are $\alpha=0$ and $C=\overrightarrow{0}$. If either $(\alpha=\beta$ and $C=D)$ or $(\alpha=-\beta$ and $C=-D)$, then (A.1) gives $e^{2 \pi i\langle z, \lambda\rangle}=-1$ which is a measure zero condition.

Otherwise, assume $\alpha=\beta$ and $C=-D$ (the other case is treated similarly). Here we have

$$
\alpha+i\langle C, \lambda\rangle=-e^{2 \pi i\langle\lambda, z\rangle}(\alpha-i\langle C, \lambda\rangle) .
$$

If $\alpha=0$ and $C \neq 0$ then there is some $\lambda \in \Lambda$ so that $\langle C, \lambda\rangle \neq 0$ and (A.7) forces $e^{2 \pi i\langle z, \lambda\rangle}=1$, that is $z$ lies on one of the hyperplanes

$$
\cup_{\lambda \in \Lambda}\{z:\langle\lambda, z\rangle=0 \bmod 1\}
$$

which is a measure zero condition.

If $\alpha \neq 0$, we replace $C$ by $-\frac{1}{\alpha} C$ and $\alpha$ by 1 and drop the negative sign. Taking the real part of (A.7), we have

$$
1+\cos 2 \pi\langle\lambda, z\rangle=\langle C, \lambda\rangle \sin 2 \pi\langle\lambda, z\rangle .
$$

We may assume that the sine on the RHS doesn't vanish, since $\sin 2 \pi\langle\lambda, z\rangle=$ 0 is a measure zero condition. Therefore, we may divide to get

$$
\langle C, \lambda\rangle=\frac{1+\cos 2 \pi\langle\lambda, z\rangle}{\sin 2 \pi\langle\lambda, z\rangle}=\cot \pi\langle\lambda, z\rangle \text {. }
$$

Now square and average the result over an orbit $\mathcal{O} \subset \Lambda$ of the group $W_{d}$ of all permutations and sign changes of the coordinates. The LHS gives

$$
\frac{1}{|\mathcal{O}|} \sum_{\lambda \in \mathcal{O}}\langle C, \lambda\rangle^{2}=\frac{E}{d}|C|^{2}
$$

by (2.3), which is independent of the orbit chosen. The RHS gives

$$
\frac{1}{|\mathcal{O}|} \sum_{\lambda \in \mathcal{O}} \cot ^{2} \pi\langle\lambda, z\rangle=1+\frac{1}{\mathcal{O}} \sum_{\lambda \in \mathcal{O}} \frac{1}{(\sin \pi\langle\lambda, z\rangle)^{2}}
$$

that is we find

$$
\frac{E}{d}|C|^{2}-1=\frac{1}{|\mathcal{O}|} \sum_{\lambda \in \mathcal{O}} \frac{1}{(\sin \pi\langle\lambda, z\rangle)^{2}} .
$$

Since $1 /(\sin \pi\langle\lambda, z\rangle)^{2}$ is even, we get the same term for $\lambda$ and $-\lambda$ and so we may replace the average over $\mathcal{O}$ by the average over $\mathcal{O} / \pm$ where we have taken only one of $\lambda,-\lambda$. Thus

$$
\frac{E}{d}|C|^{2}-1=\frac{1}{|\mathcal{O} / \pm|} \sum_{\lambda \in \mathcal{O} / \pm} \frac{1}{(\sin \pi\langle\lambda, z\rangle)^{2}}
$$

Assuming that $\mathcal{N}>\left|W_{d}\right|=2^{d} d$ !, we can find a different orbit $\mathcal{O}^{\prime} \subset \Lambda$ and then comparing with (A.8) gives

$$
\frac{1}{|\mathcal{O} / \pm|} \sum_{\lambda \in \mathcal{O} / \pm} \frac{1}{(\sin \pi\langle\lambda, z\rangle)^{2}}=\frac{1}{\left|\mathcal{O}^{\prime} / \pm\right|} \sum_{\lambda \in \mathcal{O}^{\prime} / \pm} \frac{1}{(\sin \pi\langle\lambda, z\rangle)^{2}}
$$

that is we have eliminated the variable $C$.

We claim that (A.9) forces the point $z$ to lie on a measure zero subset of $\mathbb{T}^{d}$. Indeed, the functions involved are meromorphic in $\mathbb{C}^{d} / \mathbb{Z}^{d}$ and hence if (A.9) does not hold for all $z$, it can only hold on a complex submanifold of 
codimension (at least) one and in particular its real points will have codimension at least one in $\mathbb{T}^{d}$. But near the origin $z=0$, each of the functions $1 /(\sin \pi\langle\lambda, z\rangle)^{2}$ has singularities on the hyperplane $\langle z, \lambda\rangle=0$ and these hyperplanes are distinct for $\lambda$ 's which are not collinear (here the condition $d \geq 2$ comes in), as is the case for those appearing in (A.9). Thus these functions are linearly independent and so (A.9) is not valid for all $z$.

\section{REFERENCES}

[1] Bérard, P. Volume des ensembles nodaux des fonctions propres du laplacien. BonySjostrand-Meyer seminar, 1984-1985, Exp. No. 14 , 10 pp., École Polytech., Palaiseau, 1985.

[2] Berry, M. V. Regular and irregular semiclassical wavefunctions. J. Phys. A 10 (1977), no. 12, 2083-2091.

[3] Berry, M. V. Statistics of nodal lines and points in chaotic quantum billiards: perimeter corrections, fluctuations, curvature J.Phys.A 35 (2002), 3025-3038.

[4] J. Brüning Über Knoten Eigenfunktionen des Laplace-Beltrami Operators, Math. Z. 158 (1978), 15-21.

[5] J. Brüning and D. Gromes Über die Länge der Knotenlinien schwingender Membranen, Math. Z. 124 (1972), 79-82.

[6] S. Y. Cheng, Eigenfunctions and nodal sets, Comm. Math. Helv. 51 (1976), 43-55.

[7] H. Donnelly, and C. Fefferman Nodal sets of eigenfunctions on Riemannian manifolds, Invent. Math. 93 (1988), 161-183.

[8] H. Federer, Curvature measures. Trans. Amer. Math. Soc. 931959 418-491.

[9] M. Kac, On the average number of real roots of a random algebraic equation. Bull. Amer. Math. Soc. 49, (1943), 314-320. Correction, ibid. 49, (1943) 938.

[10] J. Neuheisel, The asymptotic distribution of nodal sets on spheres, Johns Hopkins Ph.D. thesis (2000).

[11] F. Oravecz, Z. Rudnick and I. Wigman, The Leray measure of nodal sets for random eigenfunctions on the torus, math-ph/0609072, to appear in Annales de l'Institut Fourier 57, (2007).

[12] S. Zelditch Complex zeros of real ergodic eigenfunctions, Invent. Math. 167 (2007), 419-443.

School of Mathematical Sciences, Tel Aviv University, Tel Aviv 69978, ISRAEL

E-mail address: rudnick@post.tau.ac.il

Department of Mathematics and Statistics, McGill University and Centre DE ReCherches mathématiques (CRM), Université de Montréal C.P. 6128, SuCC. Centre-ville Montréal, QuéBec H3C 3J7, CANAdA

E-mail address: wigman@crm.umontreal.ca 